\title{
AIP
}

\section{Adhesive bondline interrogation using Stoneley wave methods}

Richard O. Claus and Ronald A. Kline

Citation: Journal of Applied Physics 50, 8066 (1979); doi: 10.1063/1.325943

View online: http://dx.doi.org/10.1063/1.325943

View Table of Contents: http://scitation.aip.org/content/aip/journal/jap/50/12?ver=pdfcov

Published by the AIP Publishing

\section{Articles you may be interested in}

Deformation and flow of matter: Interrogating the physics of materials using rheological methodsa)

J. Rheol. 56, 113 (2012); 10.1122/1.3671401

Time-domain solutions for Rayleigh and Stoneley waves using the single-scattering parabolic equation method.

J. Acoust. Soc. Am. 124, 2585 (2008); 10.1121/1.4783194

BONDLINE INSPECTION OF COMPLEX STRUCTURES USING GUIDED WAVES

AIP Conf. Proc. 975, 207 (2008); 10.1063/1.2902660

Nonlinear Stoneley and Scholte waves

J. Acoust. Soc. Am. 106, 1712 (1999); 10.1121/1.427920

Use of Stoneley Waves to Determine the Shear Velocity in Ocean Sediments

J. Acoust. Soc. Am. 36, 1595 (1964); 10.1121/1.1919250

MIT LINCOLN

LABORATORY CAREERS

Discover the satisfaction of innovation and service

to the nation
- Space Control

- Air \& Missile Defense

- Communications Systems \& Cyber Security

- Intelligence, Surveillance and Reconnaissance Systems

- Advanced
Electronics
- Tactical Systems
- Homeland
Protection
- Air Traffic Control

- Air Traffic Control

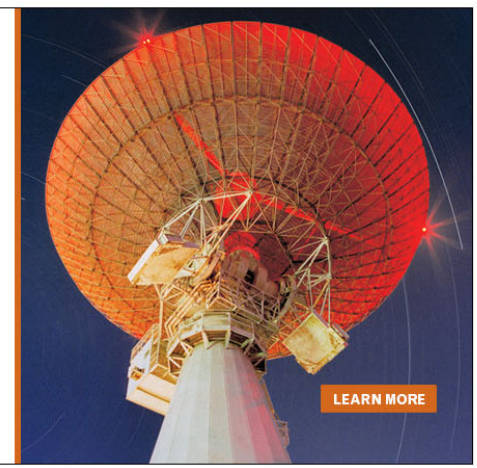




\title{
Adhesive bondline interrogation using Stoneley wave methods
}

\author{
Richard O. Claus \\ Department of Electrical Engineering, Virginia Polytechnic Institute and State University, Blacksburg, Virginia 24061
}

Ronald A. Kline

General Dynamics Fort Worth Division, Fort Worth, Texas 76101

(Received 7 May 1979; accepted for publication 27 June 1979)

\begin{abstract}
In this work, a new technique for analyzing interfacial conditions in completed adhesive bonds is discussed. This method is based on the sensitivity of Stoneley waves, which propagate along the boundary between dissimilar solid media, to changes in the material properties of the interface region. Stoneley wave attenuation measured after processing was found to increase as a function of increasing surface roughness in specimens of borosilicate crown glass bonded with an aerobic cement to a substrate of 7740 Pyrex mirror glass. Possible extensions of these results to highstrength structural adhesively bonded composites are discussed.
\end{abstract}

PACS numbers: $68.48 .+\mathrm{f}, 68.25 .+\mathrm{j}$

\section{INTRODUCTION}

The development of a nondestructive technique for post process assessment of bond strength in adhesively bonded materials has long been recognized as critical to the acceptance of adhesive bonding as a substitute for conventional fastening techniques in high-strength applications. While adhesive joining techniques offer numerous design advantages over conventional methods, there is a great reluctance to use a method which is quite sensitive to processing variables (surface treatment, cure cycle, etc.) and impossible to reliably inspect once the bonding process is completed. While large-scale defects such as voids or delamination can be detected with standard nondestructive testing procedures (ultrasonic, radiography, acoustic resonance), there is no known way to treat the problem of the "weak bond" where adhesive and adherend are in intimate contact but there is little interfacial strength. It has been proposed that in the absence of such a method, adhesive bonding could be used if the bonds were made in an environment where each step could be tightly controlled. This approach is clearly less desirable than post process inspection, as the human error factor has been neglected as well as the fact that once such parts were fabricated and in service, there would still be no way to ensure their continuing reliability.

This research is directed toward the application of experimental interfacial wave techniques to the development of a nondestructive test method for bond strength analysis in completed adhesive bonds. Many investigators have attempted to apply conventional sonic techniques to study weakly bonded adhesive systems without dramatic success. This is primarily due to the incompatibility of the wavelength $(0-100 \mu \mathrm{m})$ of normally incident bulk waves at experimentally feasible frequencies with the dimensions of the adhesive-adherend interface region $(0-100 \AA)$. Stoneley waves, on the other hand, propagate along the boundary and are not subject to this restriction. Changes in the properties of the boundary region should result in altering the characteristics of propagating Stoneley waves via attenuation, dispersion, etc. This investigation studies the effect of surface roughness on the propagation of Stoneley waves in adhesively bonded materials. An optical detection system is used for absolute measurement of the wave amplitudes of SAW impinging on and emerging from an adhesive bondline. Since this is a noncontact method, it avoids the difficulties in attenuation measurement introduced by variations in coupling efficiency when contact sensors are used.

\section{THEORY}

The first-order Stoneley theory assumes two semi-infinite isotropic elastic solids in perfect contact along the $x-y$ plane as shown in Fig. 1. ${ }^{1}$ The material constants of the media defined by $z>0$ and $z<0$ are the Lamé constants $\mu_{1}$ and $\mu_{2}$, and $\lambda_{1}$ and $\lambda_{2}$, and the densities $\rho_{1}$ and $\rho_{2}$, respectively. Induced particle displacements in the upper medium are denoted by $x_{1}, y_{1}$, and $z_{1}$ parallel to the $x, y$, and $z$ coordinate axes, and by $x_{2}, y_{2}$, and $z_{2}$, in the lower medium. Stoneley and others have shown that if a wave propagates in the positive $x$ direction along the boundary, particle motion is confined to the $x-z$ plane and has components $x_{1}, z_{1}$, and $x_{2}, z_{2}$ on either side of the boundary. ${ }^{2,3}$

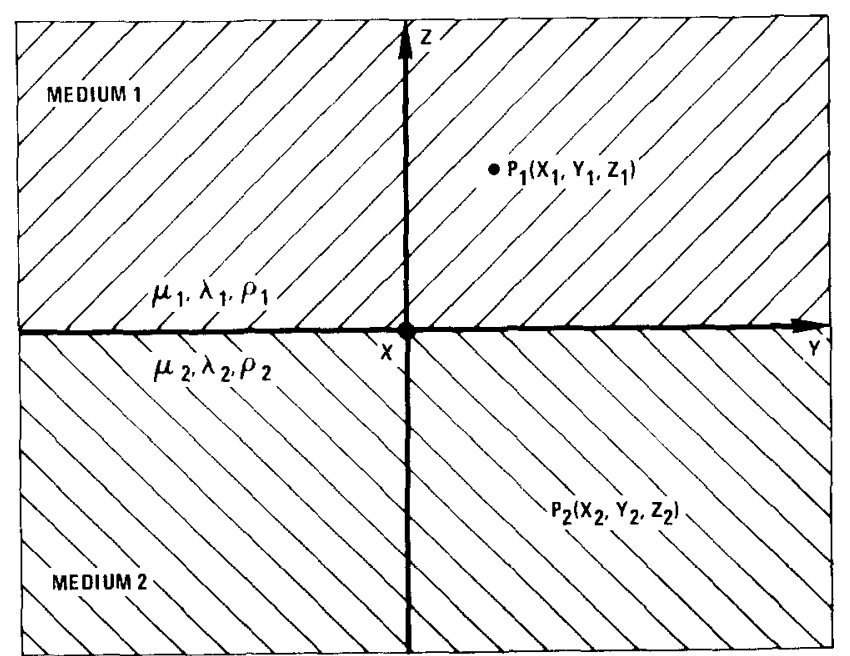

FIG. 1. Ideal Stoneley wave geometry. Infinite half-spaces $z>0$ and $z<0$ are in perfect contact at $z=0$. No relative particle motion or stress discontinuities occur at the boundary. 
Stoneley wave existence requires that both particle motion and stress components in each material be continuous across the boundary. These six individual conditions at $z=0$ may be expressed as

$$
\begin{aligned}
& x_{1}=x_{2} \\
& y_{1}=y_{2} \\
& z_{1}=z_{2} \\
& \mu_{1}\left(\frac{\partial x_{1}}{\partial z}+\frac{\partial z_{1}}{\partial x}\right)=\mu_{2}\left(\frac{\partial x_{2}}{\partial z}+\frac{\partial z_{2}}{\partial x}\right), \\
& \mu_{1}\left(\frac{\partial y_{1}}{\partial z}+\frac{\partial z_{1}}{\partial y}\right)=\mu_{2}\left(\frac{\partial y_{2}}{\partial z}+\frac{\partial z_{2}}{\partial y}\right) \\
& \lambda_{1} \Delta_{1}+2 \mu_{1} \frac{\partial z_{1}}{\partial z}=\lambda_{2} \Delta_{2}+2 \mu_{2} \frac{\partial z_{2}}{\partial z}
\end{aligned}
$$

where the dilitation $\Delta_{i}$ is defined by

$$
\Delta_{i}=\frac{\partial x_{i}}{\partial x}+\frac{\partial y_{i}}{\partial y}+\frac{\partial z_{1}}{\partial z}
$$

An elastic wave at the interface satisfying the desired stress and motion boundary conditions has the speed $C$ where

$$
\begin{aligned}
C^{4}\left[\left(\rho_{1}-\rho_{2}\right)^{2}\right. & \left.-\left(\rho_{1} M_{2}+\rho_{2} M_{1}\right)\left(\rho_{1} N_{2}+\rho_{2} N_{1}\right)\right] \\
& +2 P C^{2}\left(\rho_{1} M_{2} N_{2}-\rho_{2} M_{1} N_{1}-\rho_{1}+\rho_{2}\right) \\
& +P^{2}\left(M_{1} N_{1}-1\right)\left(M_{2} N_{2}-1\right)=0 .
\end{aligned}
$$

In Eq. (8), $M_{1} \equiv\left(1-C^{2} / \alpha_{1}^{2}\right)^{1 / 2}, M_{2} \equiv\left(1-C^{2} / \alpha_{2}^{2}\right)^{1 / 2}$, $N_{1} \equiv\left(1-C^{2} / \beta_{2}^{2}\right)^{1 / 2}, N_{2} \equiv\left(1-C^{2} / \beta_{2}^{2}\right)^{1 / 2}$, and $P \equiv 2\left(\rho_{1} \beta_{1}^{2}-\rho_{2} \beta_{2}^{2}\right)$, where $\alpha_{1}$ and $\alpha_{2}$ are the dilitational wave speeds in the two media, and $\beta_{1}$ and $\beta_{2}$ are the shear wave speeds. Equation (8) has real solutions for $C$ only if the material constants of the two bonded media are suitably related. ${ }^{4-7}$

\section{ATTENUATION MEASUREMENTS}

Experimentally, boundary conditions (1)-(6) require that no relative displacement occurs between the two media surfaces and that no voids exist at the boundary. Stress discontinuities at the interface could originate at inclusions, voids, porosity, or regions of poor adhesion. All of thse factors can affect the propagation of Stoneley waves along the boundary.

Stoneley waves were generated at 8 and $9 \mathrm{MHz}$ on several bonded sample interfaces and directly detected using a differential optical probe. As shown in Fig. 2, SAW were
TABLE I. Abrasives used to generate interface surface defects prior to bonding.

\begin{tabular}{lll}
\hline & $\begin{array}{l}\text { Nominal } \\
\text { particle } \\
\text { diameter } \\
(\mathrm{mm})\end{array}$ & $\begin{array}{l}\text { Average } \\
\text { measured } \\
\text { pit size } \\
\text { material }\end{array}$ \\
\hline $\begin{array}{l}\text { \#220 carborundum } \\
\text { \#400 carborundum }\end{array}$ & 0.025 & 0.027 \\
$\# 305$ emery & 0.064 & 0.062 \\
\hline
\end{tabular}

initially generated on the plane surface of an optically reflective 7740 Pyrex mirror glass substrate by an $x$-cut piezoelectric crystal transducer mounted on a conventional Rayleigh angle water wedge. SAW with 42 - $\AA$ peak-to-peak maximum surface particle displacement amplitudes were optically measured $1 \mathrm{~mm}$ from the top of the water wedge. At the near edge of the borosilicate crown block shown bonded to the substrate in Fig. 2, more than $95 \%$ of the energy in the incident SAW was reflected and a large SAW SWR was observed on the substrate surface between the transducer wedge and the block.

Incident SAW were mode converted at the front corner of the block into Stoneley waves on the interface between the substrate and the block as well as into dilitational and shear waves in both media. Stoneley wave attenuation from the front edge to the back edge of the block was caused in part by artificially generated interface defects. These defects were introduced on the surface of the borosilicate crown glass specimens prior to bonding by polishing the block surfaces with different grades of carborundum optical abrasive. To prevent excess wear on the edges of the $1 \times 1 \times 2 \mathrm{~cm}$ blocks, each was mounted flush with similar blocks in a 4-cm-square array and the entire array was ground on a circular 15.24$\mathrm{cm}$-diam flat mirror polishing tool. Several sets of sample blocks were uniformly ground, each set with an abrasive grit having a different diameter. Grinding produced a relatively uniform distribution of pits in the glass surface and the average diameter of these pits increased with the size of the abrasive particles. Measured average defect and abrasive sizes for the grades of abrasive used are listed in Table $\mathrm{I}^{8}$

After the sample blocks were polished, they were cleaned in dilute acid and distilled water baths, dried, and bonded to the $5.08 \times 7.62-\mathrm{cm}$ mirror glass substrates with

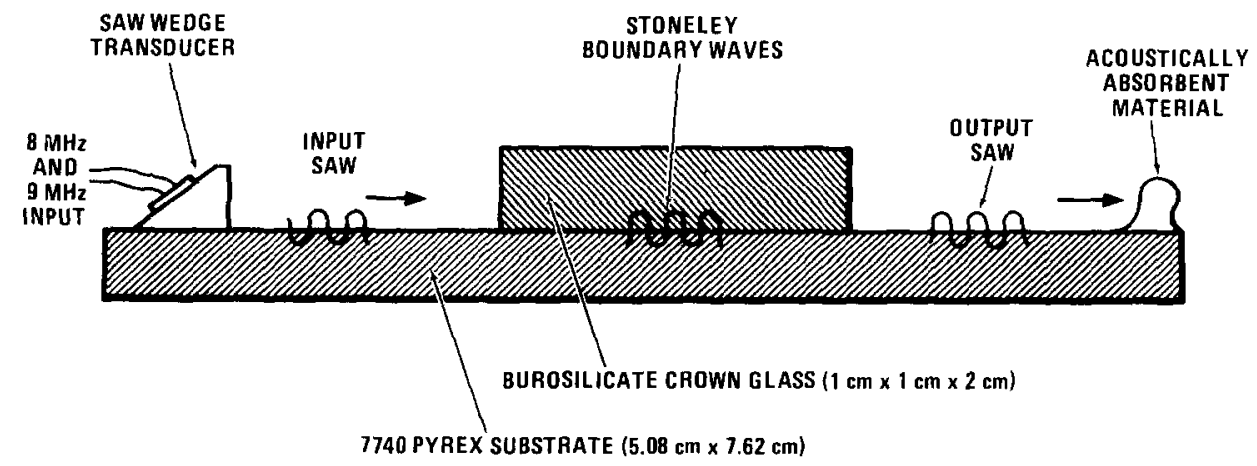

FIG. 2. SAW-to-Stoneley wave mode conversion on bonded borosilicate crown glass and Pyrex specimens. 


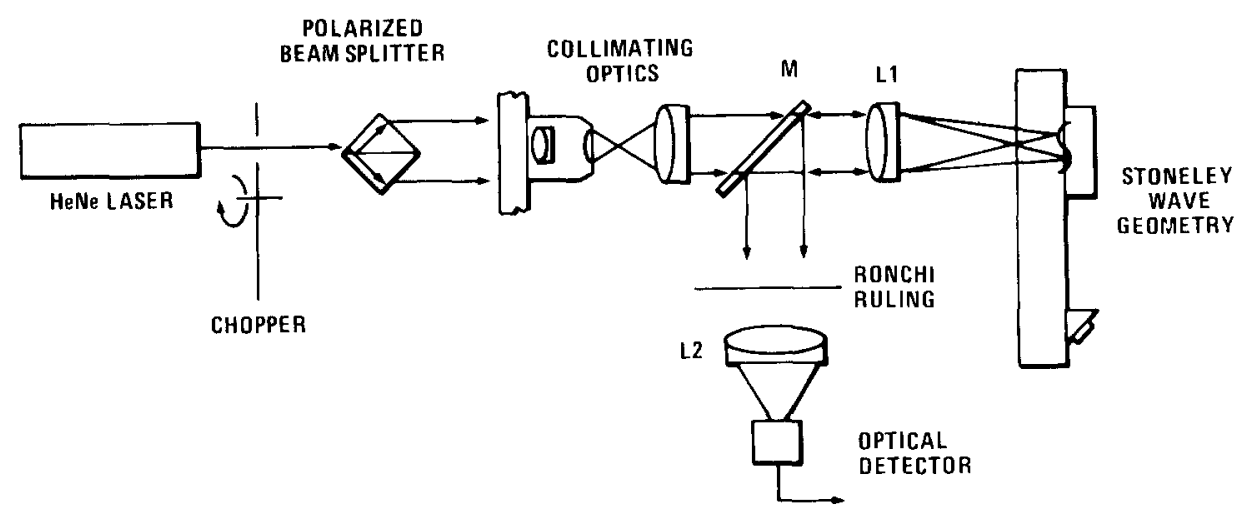

FIG. 3. Differential interferometric optics. Calibrated measurements of $3 \times 10 \quad{ }^{3} \AA$ SAW on Corning 7070 Pyrex have been obtained using this system. Light reflected from the Stoneley interface is recollimated by lens $L 1$, reflected by mirror $\mathbf{M}$ positioned below the input beams, and imaged on the Ronchi ruling. Modulation of the transmitted light is synchronously detected.

anaeorobic cement. The cemented pairs were clamped and cured for $24 \mathrm{~h}$ at room temperature. Optical inspection after curing indicated that the thickness of the bond layers had been reduced to less than $0.03 \mathrm{~mm}$, approximately $10 \%$ of the $8-\mathrm{MHz}$ Stoneley wavelength, and that no voids or gaps in the adhesive had formed.

Attenuation for each specimen pair was determined by measuring input and output SAW amplitudes as well as Stoneley-wave-induced interface particle displacements directly on the boundary. These measurements were obtained using the modified differential interferometric system shown in Fig. 3. ${ }^{9}$ On the left, the input 2.0-mW HeNe laser beam is mechanically chopped to provide a synchronous detection reference signal, divided by a polarized cube beam splitter, and expanded using a microscope objective and lens combination. The two beams are then focused as shown through the transparent glass substrate to points on the interface separated by half a Stoneley wavelength. Wave-induced particle displacements produce differential changes in the optical path lengths of the two beams. Upon reflection, the optical beams interfere producing a straight-line interference pattern that is superimposed on a straight-line Ronchi transmission grid having the same spatial period. Interface particle displacements modulate the amount of light transmitted by the grid and this light is focused on a low-noise photodetector. Detected optical signal power is approximately propor-

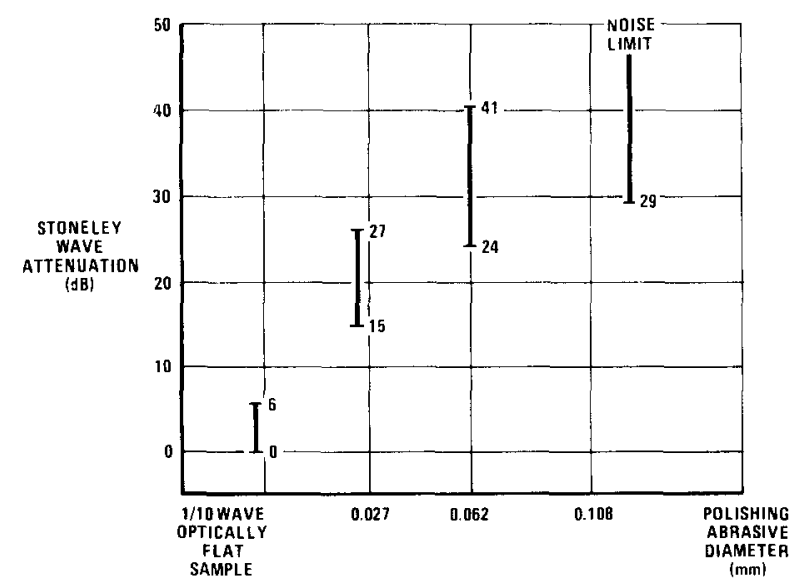

FIG. 4. Stoneley wave attenuation versus polishing abrasive diameter. Attenuation is normalized to the least attenuation measured for unpolished flat optical substrates. tional to Stoneley wave amplitude if that amplitude is $10 \AA$ or less. Absolute calibration may be obtained by comparing the theoretical and measured optical system transfer functions and by calculating the electronic system response. Calibrated measurements of $9-\mathrm{MHz}$ standing surface waves on Pyrex with peak-to-peak particle displacement of $3 \times 10^{-3}$ $\AA$ have been obtained using this system. ${ }^{10}$

\section{RESULTS}

Measured Stoneley wave attenuation is plotted in Fig. 4 versus boundary pit size. Attenuation was determined by measuring SAW amplitudes at the front and back edges of the crown glass blocks. Sets of four blocks were prepared using each of three grades of abrasive. The polished blocks as well as unpolished $1 / 10$ wave optically flat samples were bonded and the composites tested at both 8 and $9 \mathrm{MHz}$. No significant difference between 8- and 9-MHz attenuation was detected.

Probable causes of data scatter are variations in geometrical alignment between samples and nonuniform surface polishing between the individual samples in each set. ${ }^{11}$

\section{CONCLUSIONS}

The research demonstrates the potential of using Stoneley wave techniques for the evaluation of adhesively bonded materials. It is recognized that surface conditions and the properties of the interface region are the most critical factors in determining the mechanical behavior of bonded structures. Measurements of Stoneley wave attenuation as a function of boundary surface roughness indicate that the technique is sensitive to changes in interfacial conditions. These results should be extended to a more detailed study of interfacial phenomena in order to assess the applicability of this experimental method to bond strength determination in high-strength adhesive systems. Attention should be directed toward assessing the effects of chemical surface contamination on Stoneley wave propagation. It is hoped that this method will be the basis for an industrial (particularly for high-performance aircraft) evaluation system for completed adhesive bonds.

\section{ACKNOWLEDGMENT}

This work was supported in part by National Science Foundation Grants No. ENG-78-05773 and ENG-7811040. 
'R. Stoneley, Proc. R. Soc. London 106, 416 (1924).

${ }^{2}$ A.S. Ginzbarg and E. Strick, Bull. Seismol. Soc. Am. 48, 51 (1958).

${ }^{3}$ T.E. Owen, Prog. Mater. Res. 6, 69 (1964)

${ }^{4} \mathrm{~K}$. Sezawa and K. Kanai, Tokyo (Imperial) Univ. Earthquake Res. Inst.

Bull. 16, 504, (1938); 16, 683 (1938); 17, 1 (1939).

'J.G. Scholte, Proc. Ned. Akad. von Wet. 45, 20 (1942); 45, 159 (1942); 45,

439 (1942); 45, 516 (1942); 49, 120 (1946).

'T.C. Lim and M.J.P. Musgrave, Nature 225, 372 (1970)
${ }^{7}$ R.N. Thurston, J. Acoust. Soc. Am. 64, 1 (1978).

${ }^{8} \mathrm{~J}$. Strong, Procedures in Experimental Physics (Prentice-Hall, Englewood Cliffs, N.J., 1938).

${ }^{9}$ C.H. Palmer, J. Acoust. Soc. Am. 53, 948 (1973)

${ }^{10}$ C. H. Palmer, R.O. Claus, and S.E. Fick, Appl. Opt. 16, 1849 (1977).

${ }^{11}$ R.O. Claus, Proc. First Intl. Symp. on Ultrasonic Mat. Characterization Gaithersburg, Md., 1978 (unpublished) 